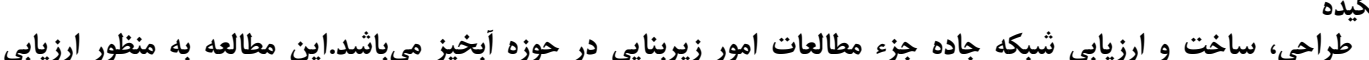

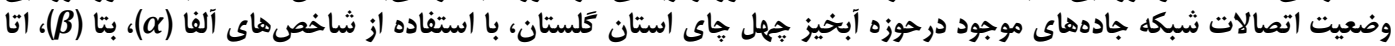

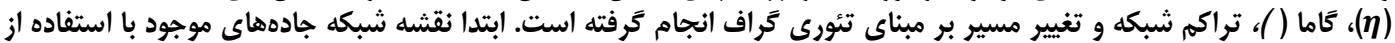

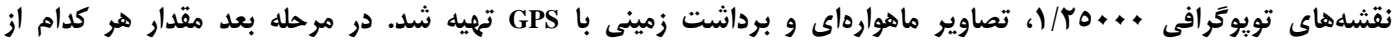

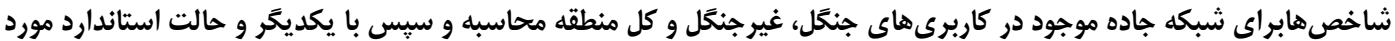

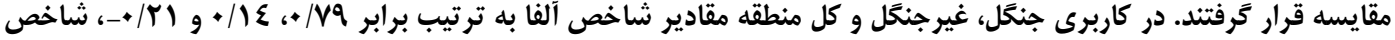

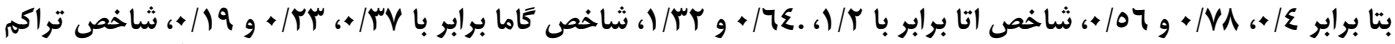

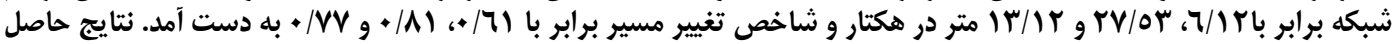

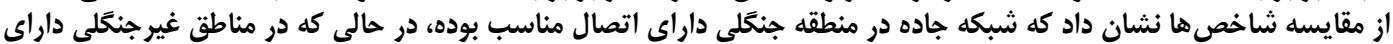

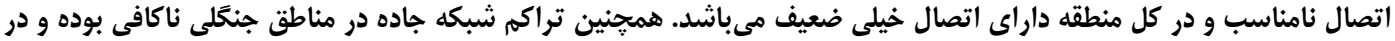

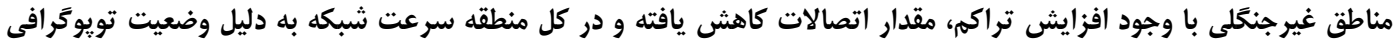

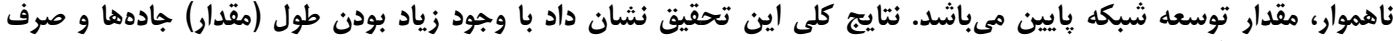

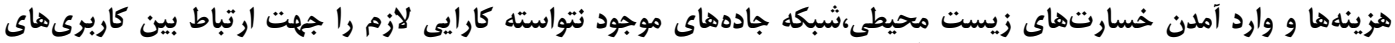

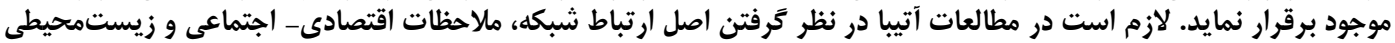

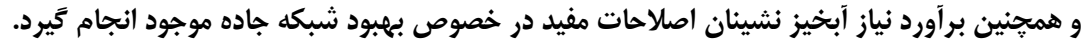

وازههاى كليدى: قطعات جاده، شاخص اتصالى، شبكهجاده، كاربرى جنكَل و غير جنكل، واحد مديريت

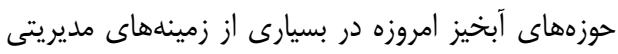

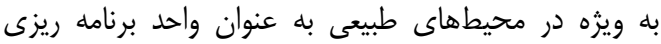

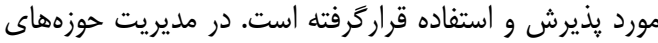

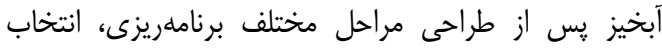

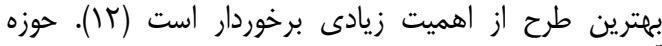

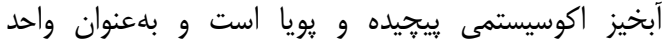

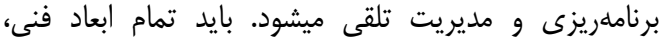

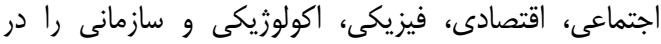

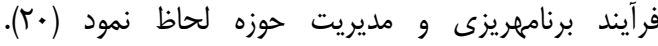

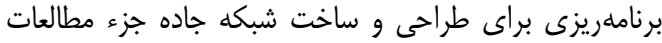
امور زيربنايى در حوزه آبخيز مى باشئ ساخد.

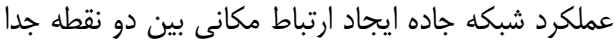

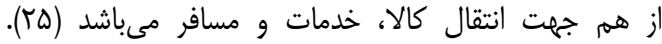

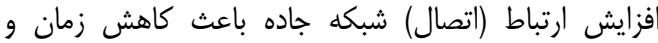

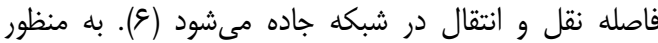

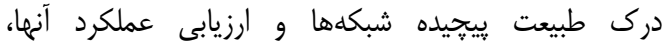

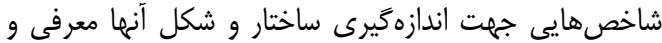

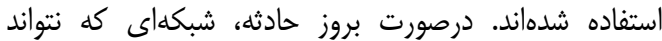

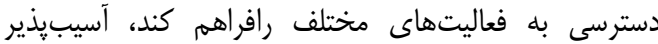

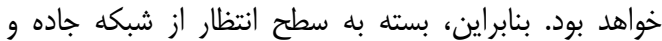
مقدار وكسترش اين فعاليتها، معيارهاى مؤثر بر بر عملكرد

شبكه نيز، متنوع خواهند بود (9).

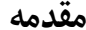

جاده به عنوان نخستين و طبيعىترين وسيله ارتباط انسان

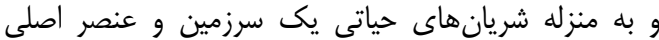

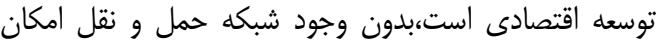

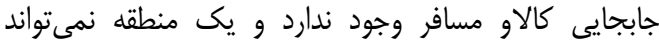

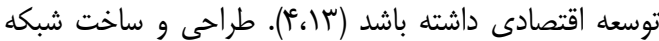

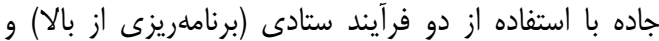

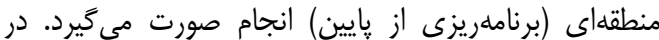

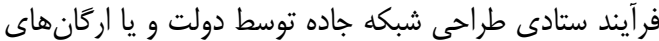

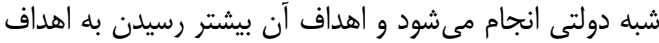

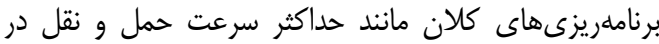

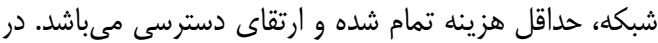

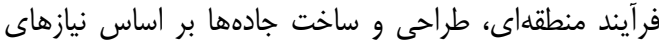

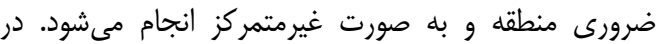

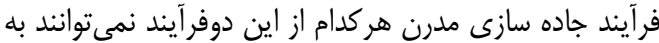

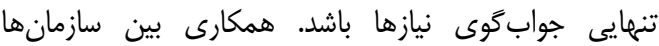

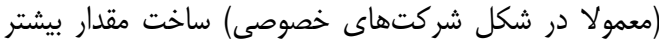

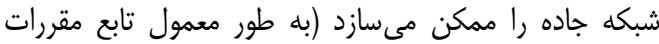

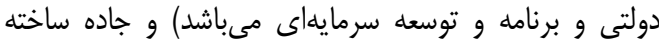
شده توسط سازمان هاى محلى بله مراتب بيشتر ازئ از مقدار ساخته شده توسط دولت مركزى است (9) مانى 


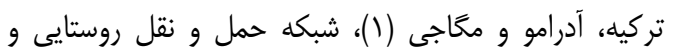

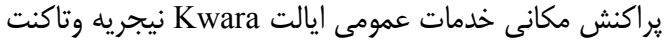

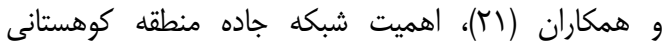
Maurienne Valley

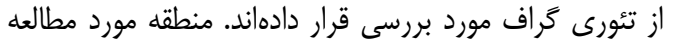

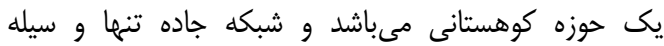

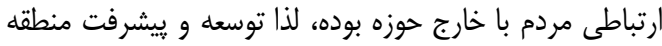

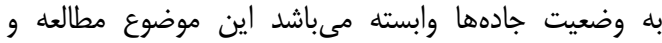

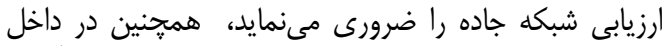

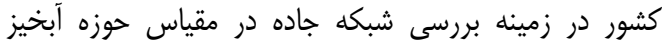

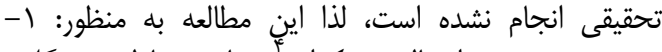

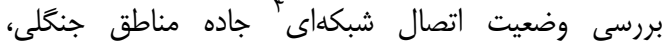

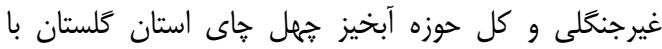

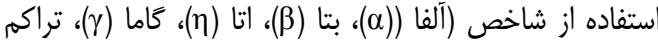

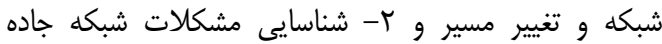

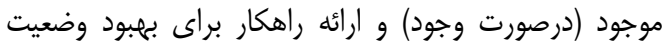

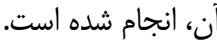

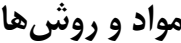 منطقه مورد مطالعه}

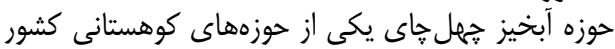

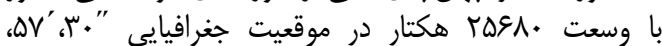

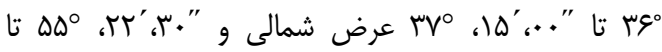

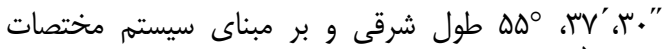

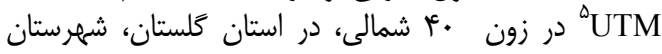

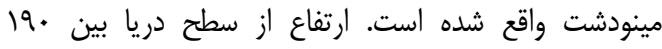

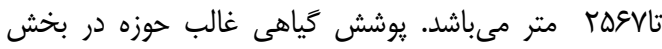

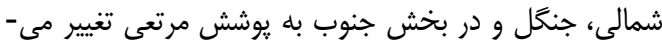

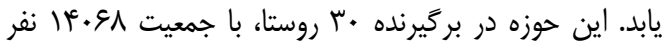

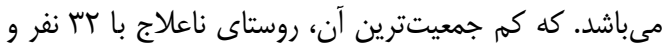

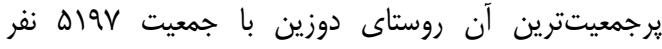

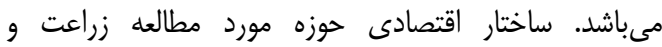

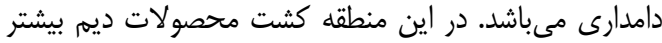

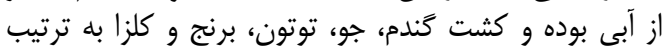

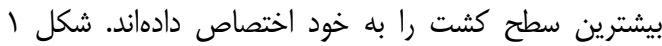

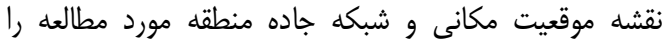

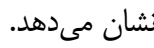

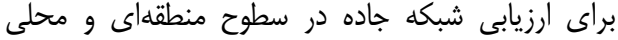

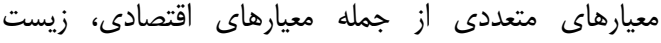

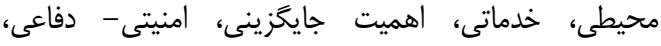

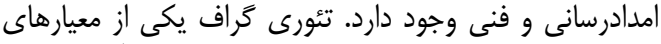

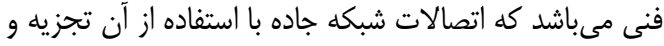

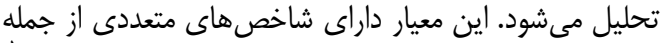

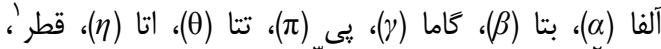

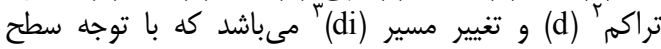

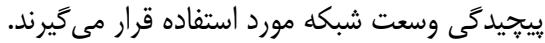

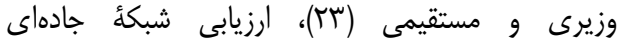
بزر كراههاى آسيا و اقيانوسيه را با استفاده از معيارهاى آلفا، بتانيا

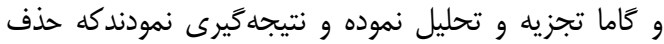

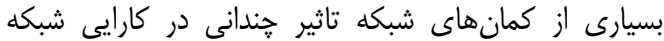

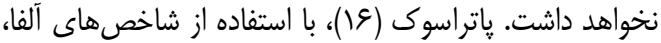

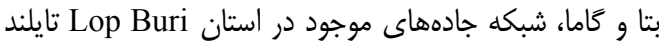

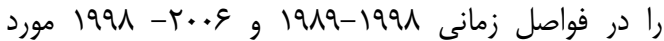

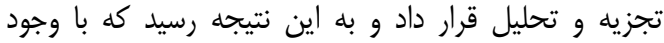
افزايش مقدار طول جاده در اين دوره زمانى مانى مقدار شاخصها

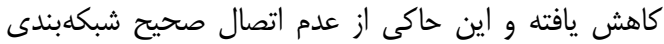

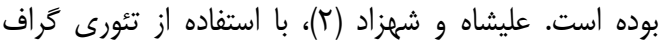

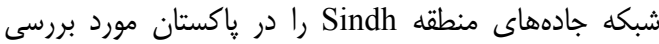

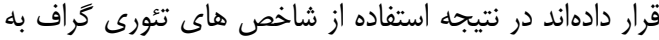

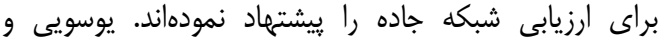

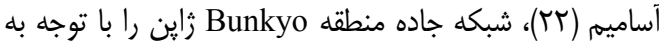

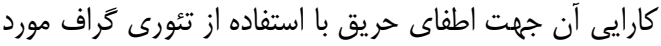

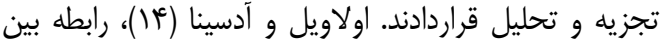

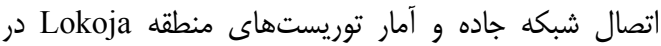

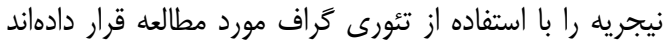

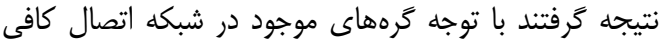

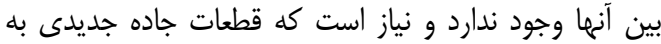

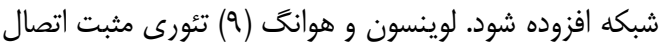

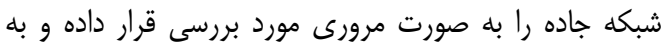

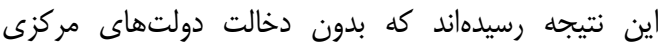

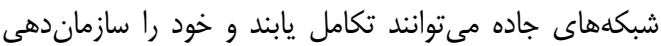

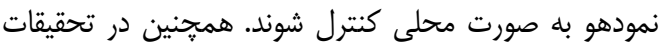

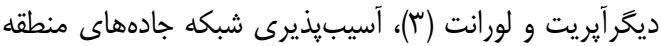

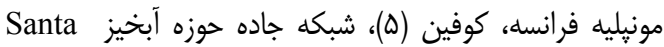
فلوريداى آمريكا، كوفى (^)، ساختار شبكه جاده استانبول

\begin{tabular}{lll}
\hline 1- Diameter & 2- Network density & 3- Detour index \\
4- Network Connectivity & 5- Universal Transverse Mercator
\end{tabular}




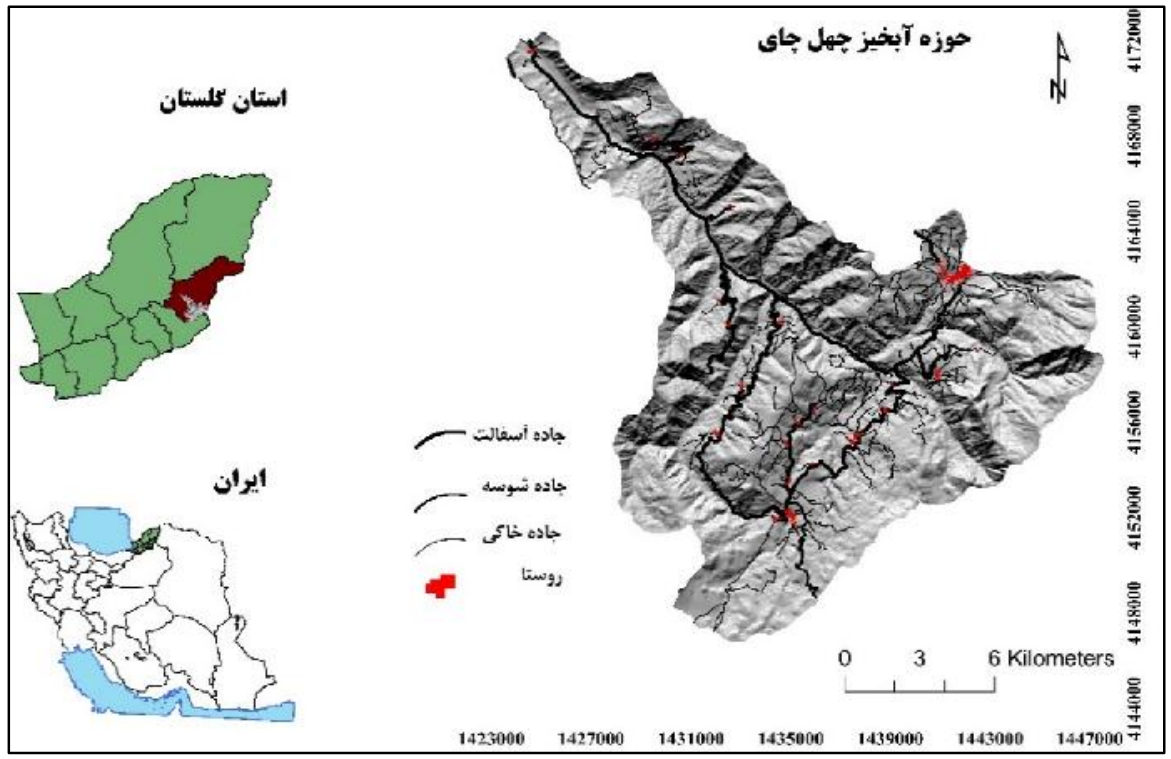

شكل ا- نقشه حوزه آبخيز جبل حاى و جادههاى موجود

Figure 1. Mape of the Chehel-chay Watershed and existing road

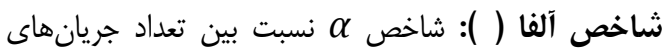

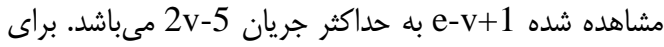

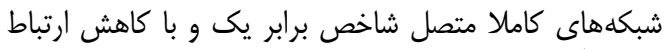

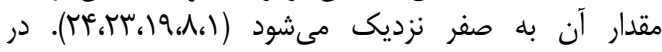

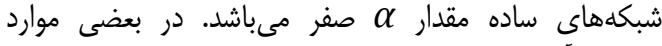

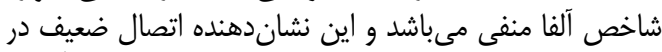

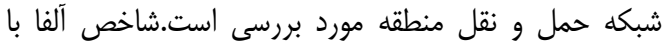

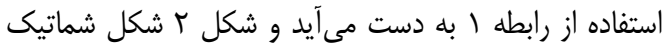
آن را نشان مىدهد.

$\alpha=\frac{e-v+1}{2 v-5}$

رابطه (1)

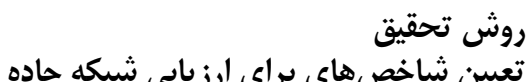

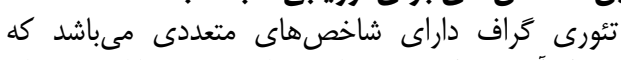

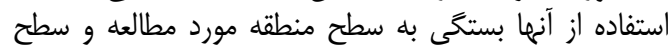

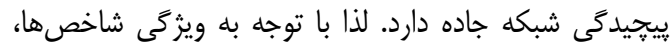

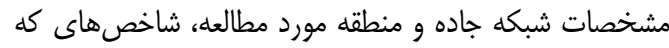

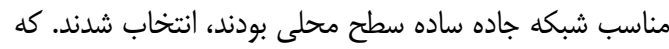

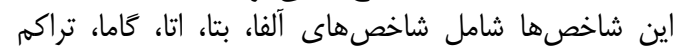
شبكه و تغيير مسير مىباشندا شارل

e= تعداد قطعات، v=تعداد كَ مها

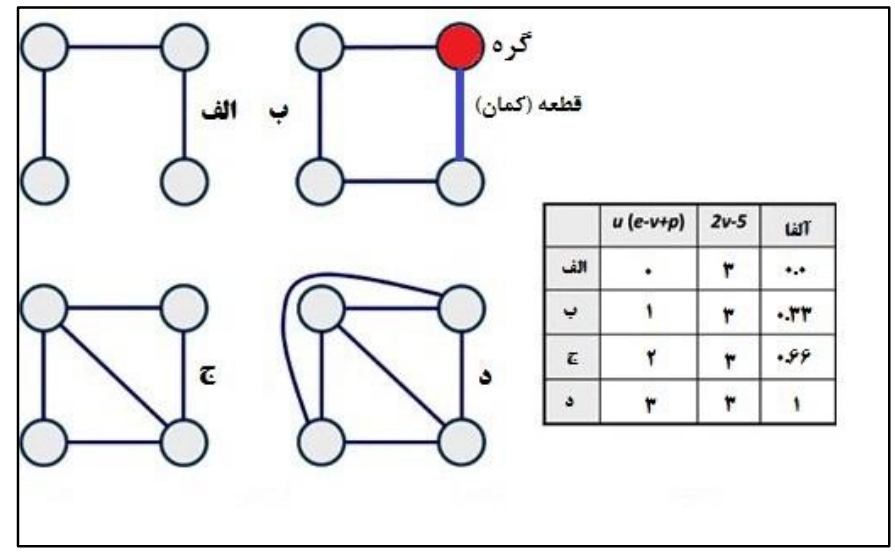

شكل r- حالت شماتيك شاخص آلفا

Figure 2. The schematic mode of Alpha index 
هند اتصال مقدار اين شاخص بيشتر از يك مى بـاشد. شاخص

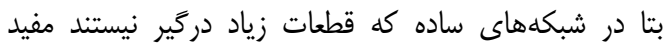

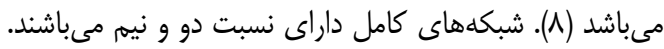

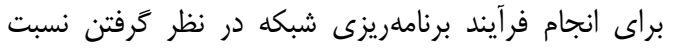

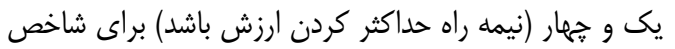

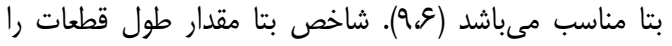

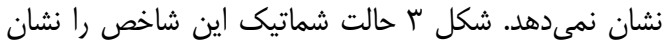

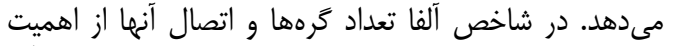

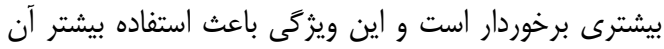

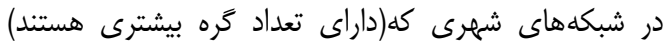

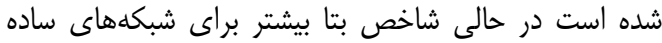

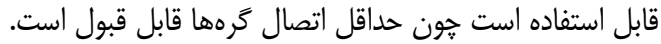

شاخص بتا (م): شاخص بتا نسبت قطعات به گره و معيار

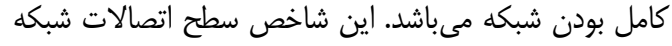

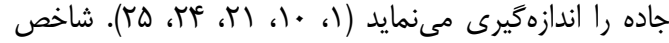

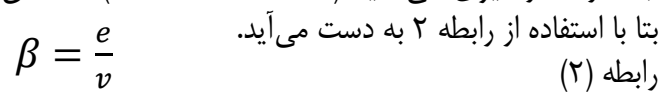
e= تعداد قطعات، v=تعداد گرهها

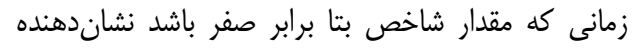

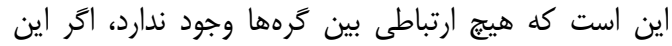

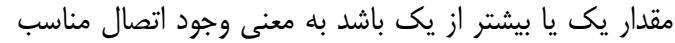

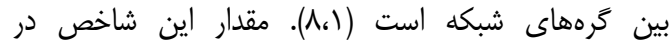

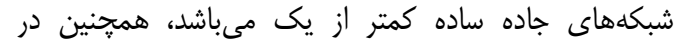

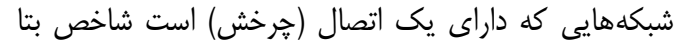

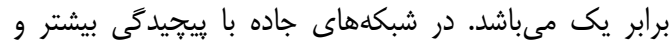

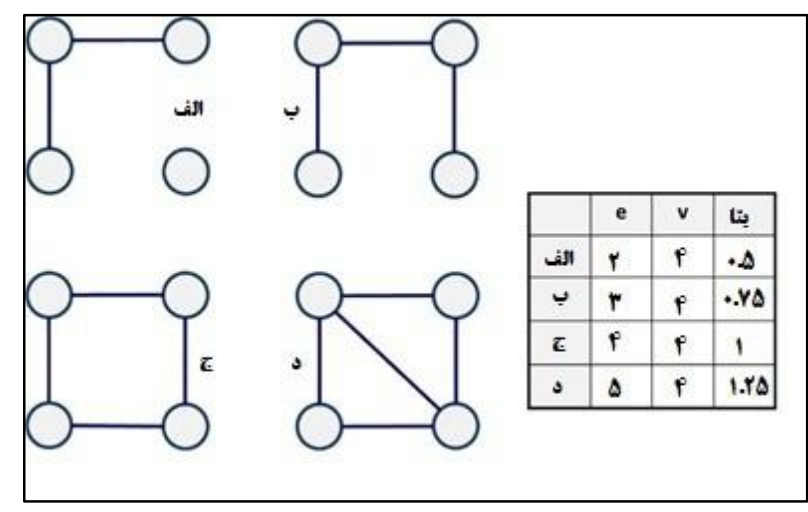

شكل س- حالت شماتيك شاخص بتا

Figure 3. The schematic mode of Beta index

نشاندهنده اتصال كامل در شبكه مىباشد و مقدار صفر به معنى اتصال ضعيف مىباشد. شاخص تغيير مسير: اندازهيرى كانديف كارايى شبكه حمل ونقل و

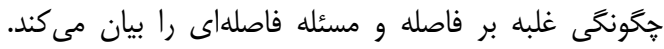
مقدار اين شاخص از نسبت فاصله افقى بله فاصله فاصله روى راصى شبكه

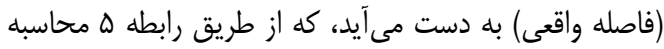

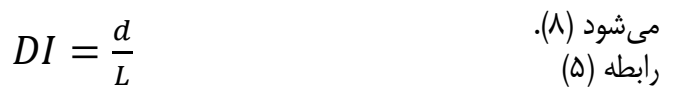

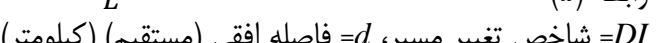

L فاصله شبكه (كيلومتر)

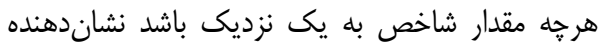

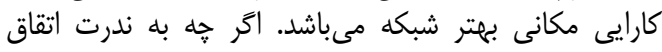

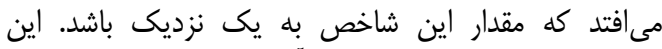
شاخص بدون واحد است و ارزش آن از صفر تا يك مئ مىباشد.
شاخص اتا (n): شاخص اتا ميانخين طول قطعات را در

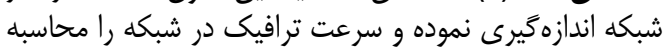

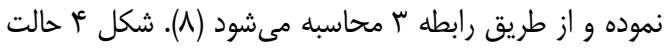
خ

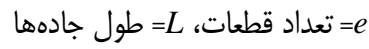

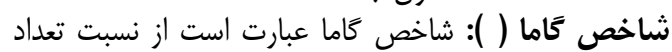

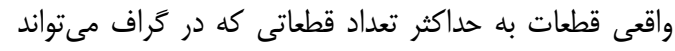

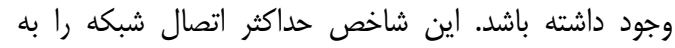

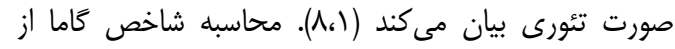
$\gamma=\frac{e}{3(v-2)}$ طريق رابطه ع محاسبه مى رئود. رابطه (أ) e= تعداد قطعات، v=تعداد گرهها

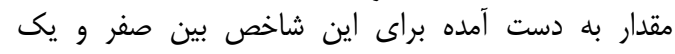

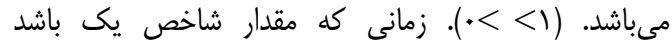




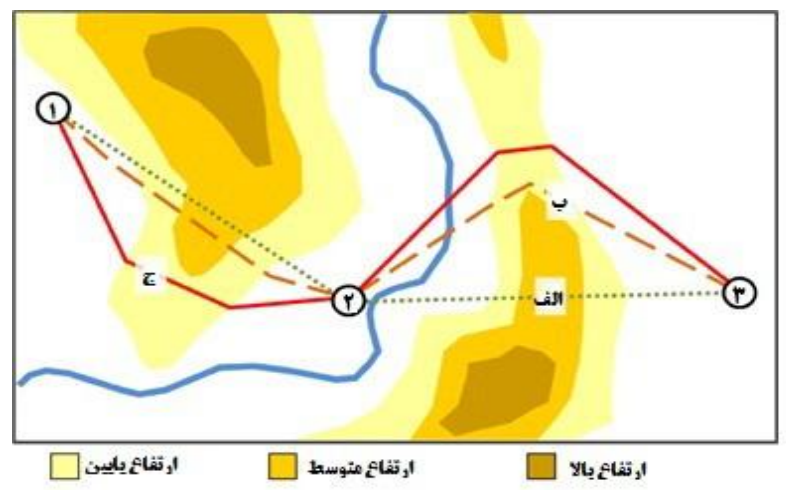

شكل ז- مسير مستعيم و انحرافى بين مسيرهاى الع، ب و ج.

Figure 4. The deviation between the straight line paths A, B and C

تعيين كَره و قطعات (كمان) هاى شبكه جاده

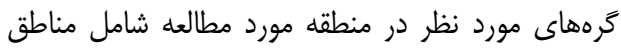

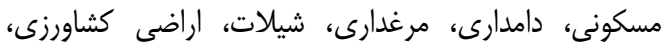

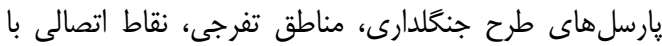

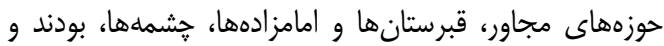

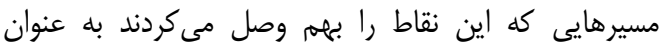

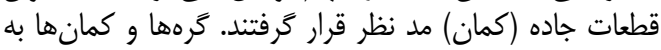

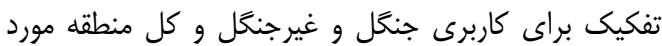

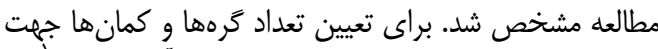

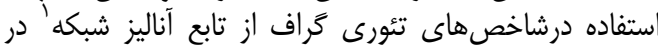

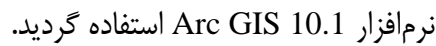

محاسبه شاخصها

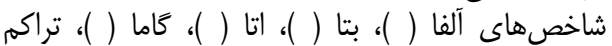

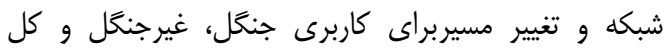

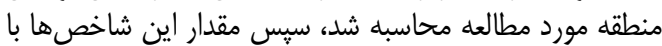

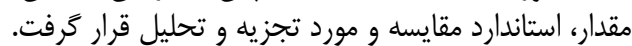

\section{نتايج و بحث}

طول و تراكم كل جادهاداى موجود

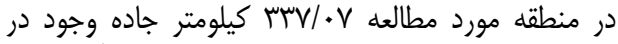

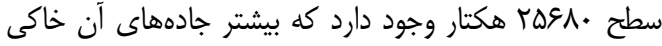

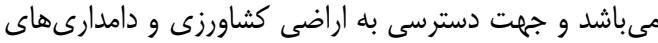

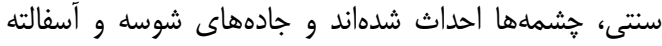

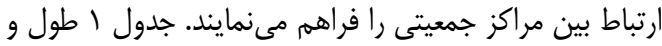

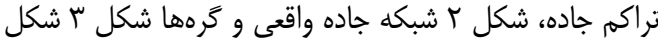

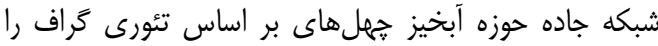
كه با استفاده از برنامه آناليز شبكه در نرمافزار

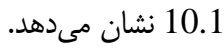

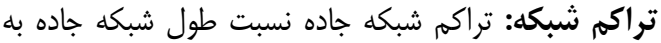

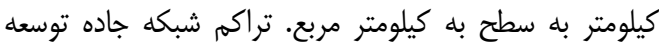

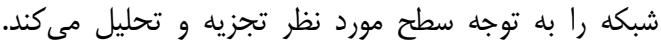

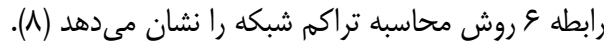
$N D=\frac{L}{A}$

رابطه (\&) ND تراكم جاده، A= مساحت منطقه مورد مطالعه (كيلومتر

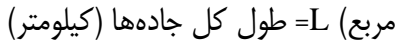
واحد تراكم براساس كيلومت جادما بكلومتر) كيلومتر مربع و يا هكتار مىباشد. تهيه نقشه شبكه جاده موجود

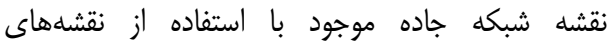

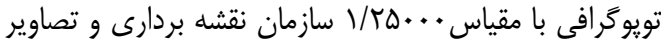

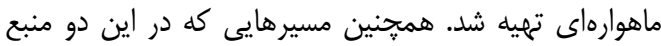

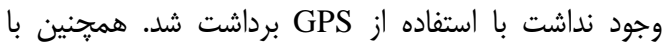

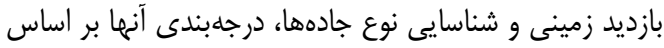

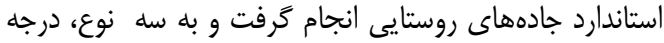
يك (آسفالت)، درجه دو (شوسان) و درجها سله سه (خاكى)

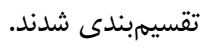

تهيه نقشه كاربرى اراضى منطقله مورد مطالعه

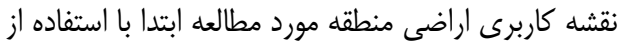

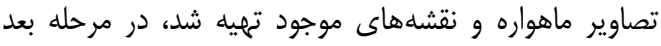

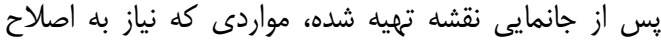

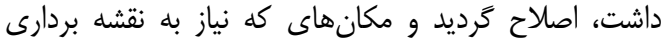

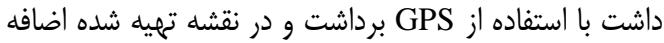

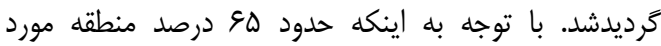

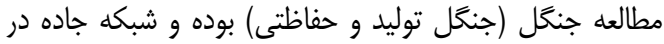

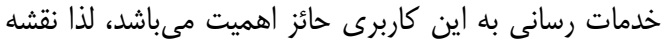

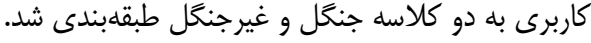



ارزيابى اتصال شبكهاى جادهاى حوزهاى آبخيز كوهستانى با استفاده از تئورى گراف در حوزه جهل جإى استان كلستان.

Table 1. Road network length and density of the Chehel-chay Watershed

جدول 1- طول و تراكم جاده موجود در حوزه آبخيز جلجلجاي

\begin{tabular}{|c|c|c|}
\hline تراكم (متر / هكتار) & طول (كيلومتر) & نوع جاده \\
\hline$r / T r$ & $\Lambda r / A V$ & آسفالت \\
\hline . & 1.199 & شوسه \\
\hline$q / 4 V$ & TFT/DF & خاكى \\
\hline 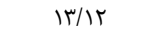 & $r \mathrm{rV} / \cdot \mathrm{V}$ & كل \\
\hline
\end{tabular}

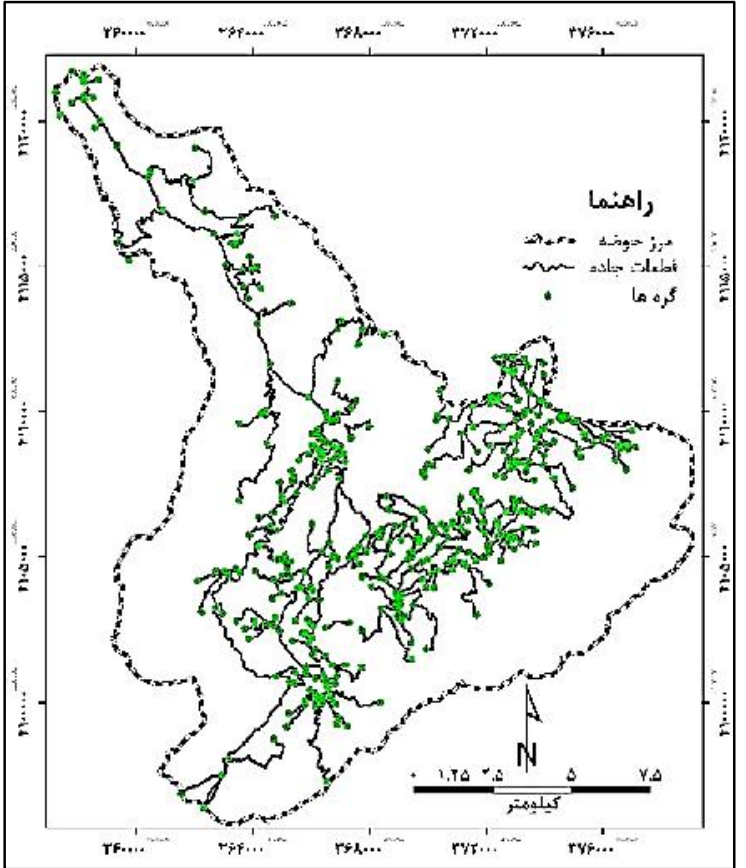

(ب)

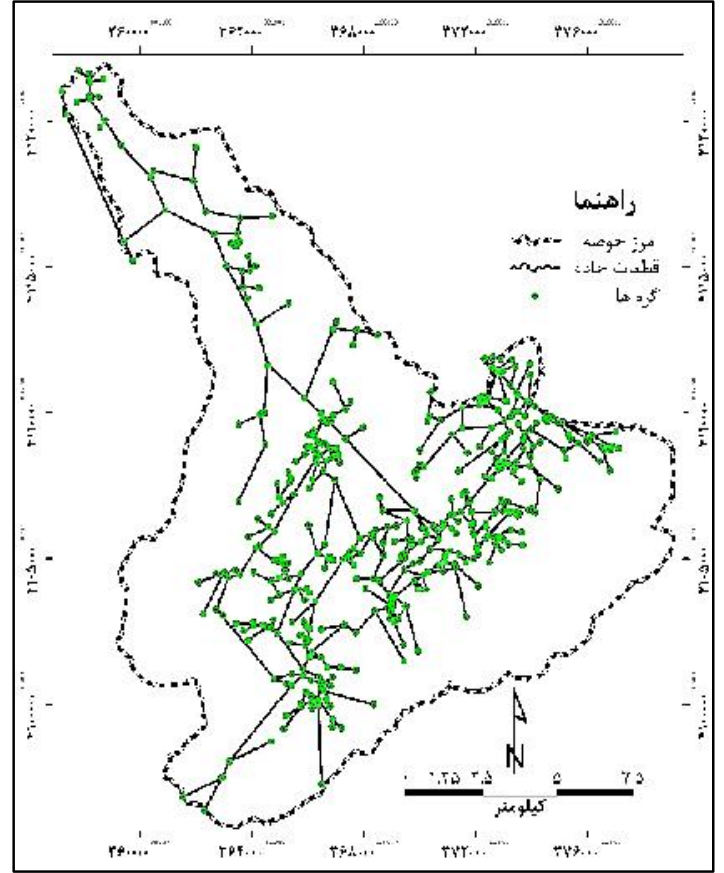

(الف)

شكل r- الف) نقشه واقعى شبكه جاده و گرهها موجود حوزه آبخيز خهل خاى ب) شبكه جاده بر اساس تئورى گراف و گرههاى موجود حوزه

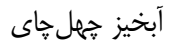

Figure 2. Existing road network (A), edges and vertices of existing road network of the Chehel-chay Watershed (B)

وكمتر بودن آن از يك نشاندهنده وضعيت تويوگر افى ناهموار

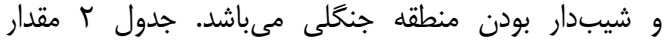

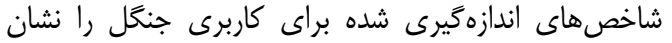

مى دهد.

\section{تجزيه و تحليل جادههاي كاربرى غيرجني}

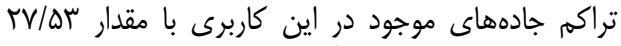

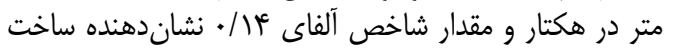

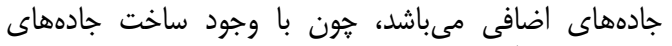

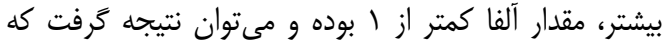

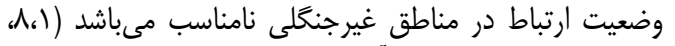

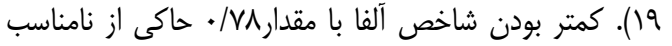

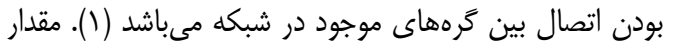

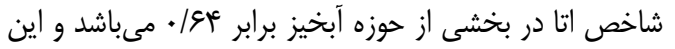

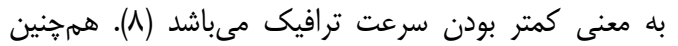

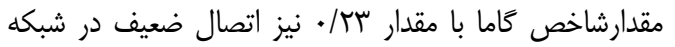

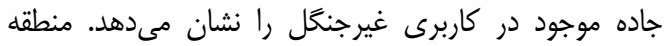

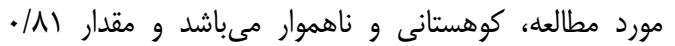
شاخص تغيير مسير در ناحيه امرى معقول به نظر مىرسل.
تجزيه و تحليل جادههاى موجود در كاربرى جنكَل

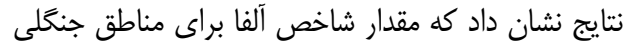

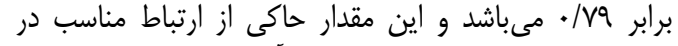

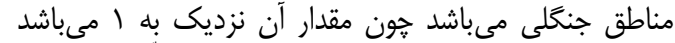
(ب)

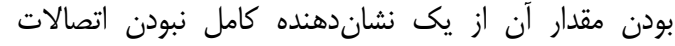

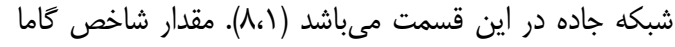

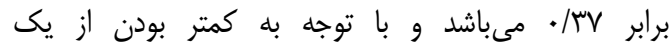

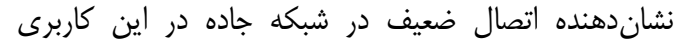

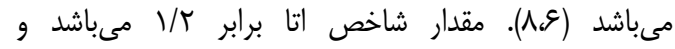

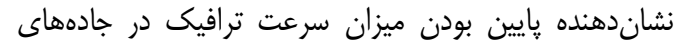

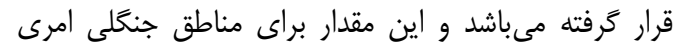

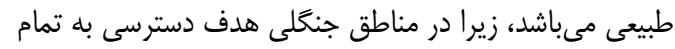

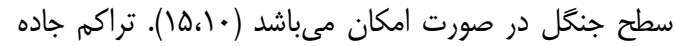

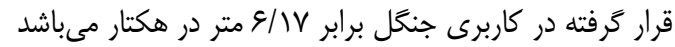

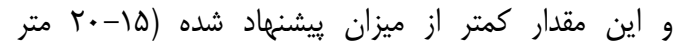
درهكتار) توسط لطفعليان و يارساخو (11) مىباشد. مقار مقدار

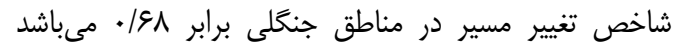




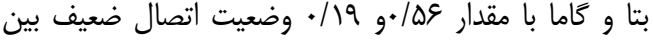

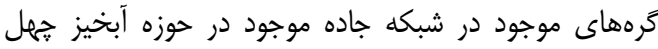

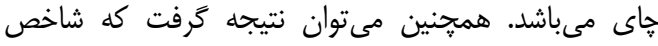

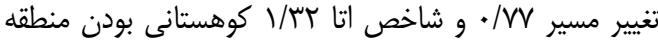

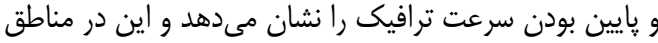

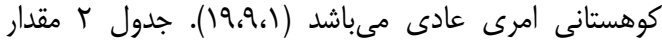

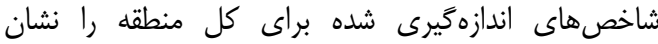
مى دهد.
جدول ץ وضعيت شاخصهاى اندازهگيرى شده در كاربرى

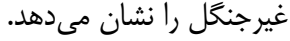

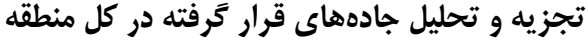

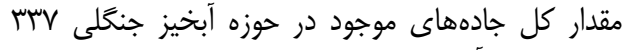

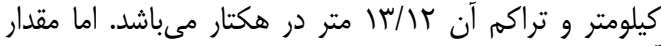

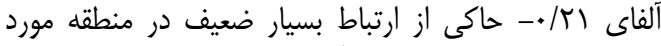

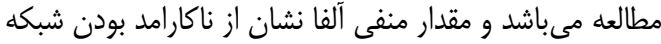

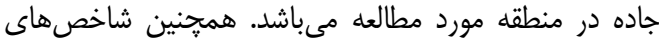

جدول r- مقدار شاخصهاى اندازهيرى شده براى كاربرى جنگل، غيرجنگل و كل حوزه آبخيز جهل جاى Table 2. The measured indicesof forest, non-forest land use and total of the Chehel-chay Watershed

\begin{tabular}{|c|c|c|c|c|c|c|}
\hline شاخص تغيير مسير & تراكم شبكه (متر /هكتار) & شاخص كاما & شاخص اتا & شاخص بتا & شاخص آلفا & نوع كاربرى \\
\hline$\cdot / 91$ & 9/IV & $\cdot / \Psi V$ & $1 / T$ & $\cdot / 4$ & $\cdot /$ / 9 & جنگل \\
\hline$\cdot|A|$ & TV/QT & שr/ & .194 & $\cdot / \mathrm{V} \Lambda$ &.$/ 1 F$ & غير جنكل \\
\hline.$/ \mathrm{Vr}$ & r & .119 & $1 / \pi r$ & $.10 \%$ &.$-|r|$ & كل منطقه \\
\hline
\end{tabular}

مسيرهاى نامناسب اصلاح و يا از شبكه جاده خارج و با انجام

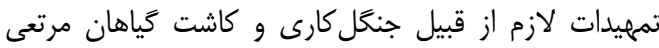

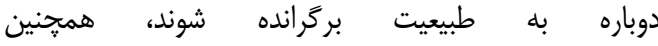

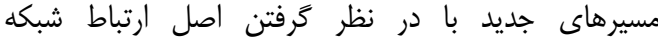
(Network Connectivity) و زيست محيطى و همجنين برآورد نياز آبخيز نشينان طراحى إنهى شود.

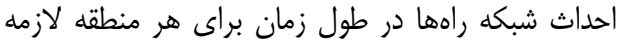

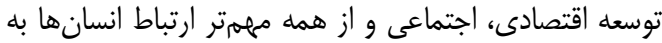

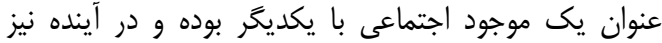

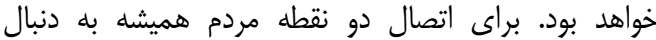

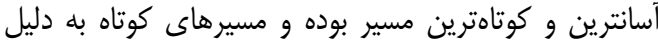

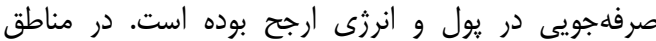

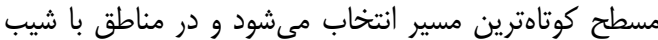

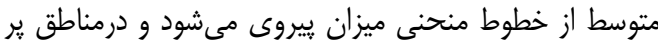

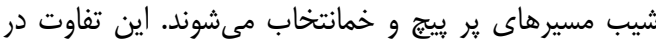

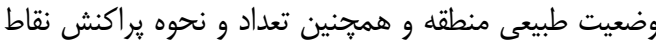

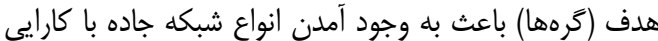

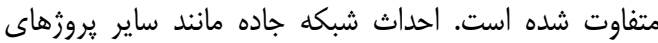

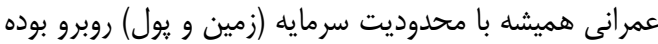

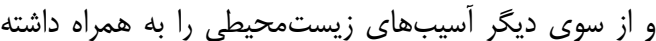

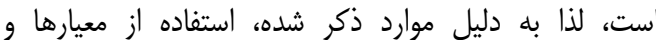

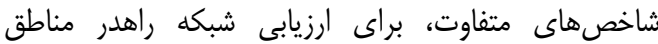

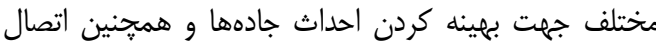

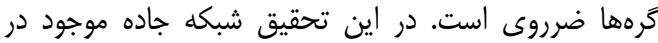

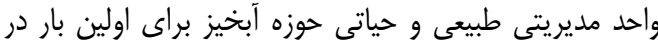

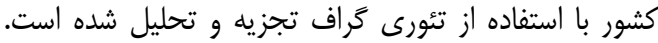

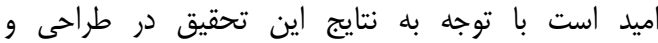

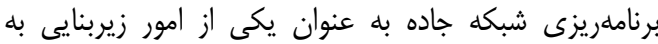

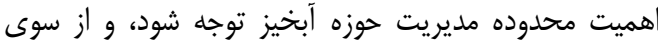

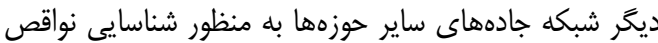

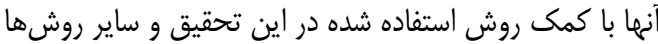

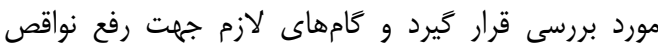

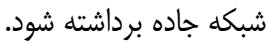

نتايج حاصل از اندازه شاخصهاى مورد اندازهيرى در داده

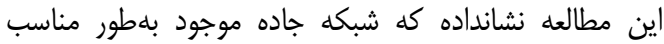

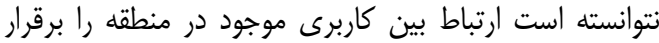

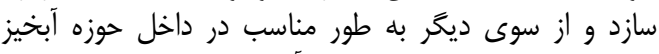

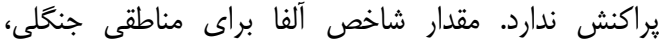

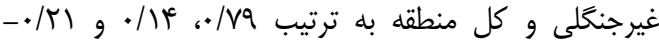

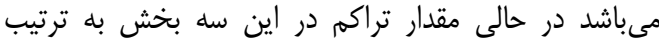

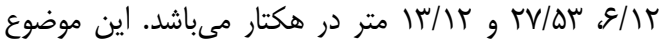

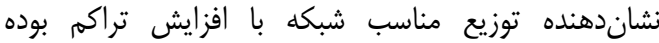

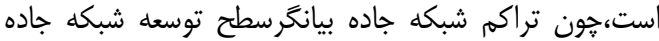

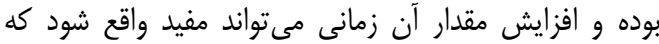

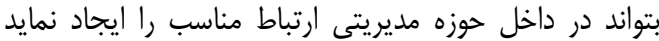

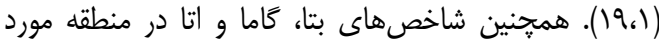

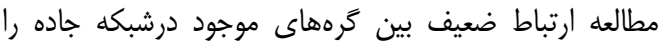

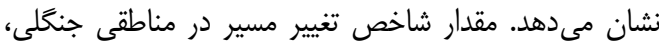

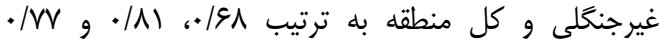

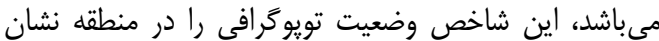

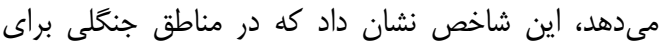

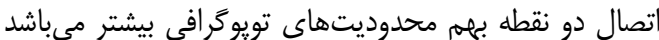

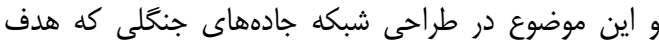

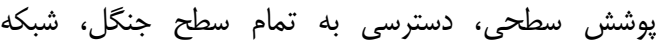

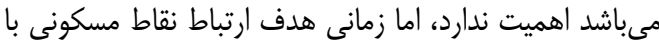

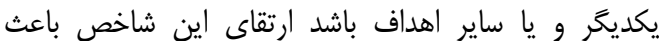

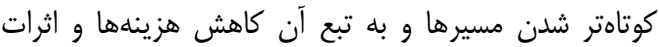

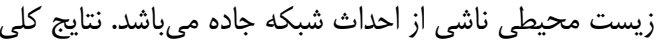

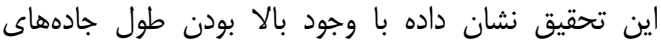

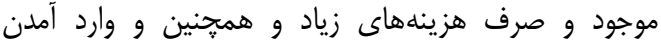

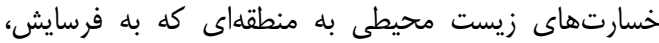

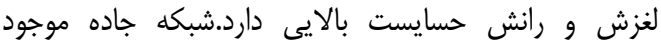

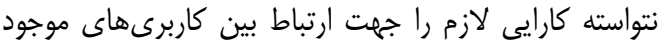

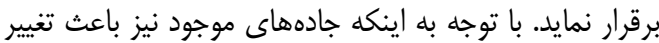

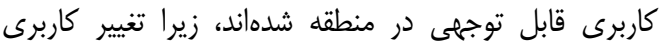

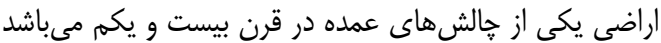
(1). لازم است درمطالعات آتى با بر رسى شبى شبكه جاده موجود 
1. Aderamo, A.J and S.A. Magaji. 2010. Rural Transportation and the Distribution of Public Facilities in Nigeria, A Case of Edu Local Government Area of Kwara State. Journal of Human Ecology, 29: 171179.

2. Ali Shah, M.A. and F. Shehzad. 2009. Evaluation of Shortest Paths in Road Network. Pakistan Journal of Commerce and Social Sciences, 3: 67-79.

3. Appert, M. and C. Laurent. 2007. Measuring urban road network vulnerability using graph theory: the case of Montpellier's road network. 23 pp.

4. Black, W.R. 2003. Transportation: A Geographical Analysis. New York: Guilford Press, 375 pp.

5. Coffin, A.W. 2009. Road network development and landscape dynamics in the Santa Fe River Watershed, North-central Florida, a dissertation presented to the graduate school of the university of Florida in partial fulfillment of the requirements for the degree of doctor of philosophy, University of Florida, $175 \mathrm{pp}$.

6. Dill, J. 2004. Measuring Network Connectivity for Bicycling and Walking. Join Congress of ACSPAESOP, Leuven, Belgium, 17 May, 20 pp.

7. Falahtabar, N. 2001. Geographic factorsimpacton the country's road network. Journal of Geographical Research, 38: 47-55 (In Persian).

8. Kofi, G.E. 2010. Network based indicators for prioritsing the location of a new urban transport connection, Case study Istanbul, Turkey, Thesis submitted to International Institute for Geoinformation Science and Earth Observation, Specialization, (Urban planning and Management). 101 pp.

9. Levinson, D. and A. Huang. 2012. A positive theory of network connectivity. Environment and Planning B: Planning and Design, 39: 308-325.

10. Lotfalian, M. 2011. Wood Transportation. Aeezh press, $342 \mathrm{pp}$ (In Persian).

11. Lotfalian, M. and A. Parsakhoo. 2012. Forest road Network Planning. Aeezh press, 155p. (In Persian).

12. Naderi, N., M. Mohseni Saravi, A. Mlakian and D. Ghasemian. 2011. AHP is a technique for decision making in Watershed. Journal of Environment and Development, 2: 41-50 (In Persian).

13. Narimani, G. 2007. Geometric Design of Highways. University of Tehran press, 584p. (In Persian).

14. Olawale, T.N. and K.I. Adesina. 2011. An Assessment of the Relationship between Road Network Connectivity and Tourists' Patronage in Lokoja Metropolis, KogiState. Journal of Natural Sciences Research, 3: 1-11.

15. Parsakhoo, A. 2015. Forest road construction and maintenance, 243 pp (In Persian).

16. Patarasuk, P. 2013. Road network connectivity and land-cover dynamics in Lop Buri Province, Thailand. Journal of Transport Geography, 28: 111-123.

17. Puu, T. 2003. Mathematical Location and Land Use Theory: An Introduction. Berlin: Springer, 344pp.

18. Rahmani, N., K. Shahedi, K. Soleimani and M. Miryaghoubzadeh. 2016. Evaluation of the Land use Change Impact on Hvdrologic Characteristics (Case Study: Kasilian Watershed). Journal of watershed management research.7: 23-33 (In Persian).

19. Rodrigue, J., C. Comtois and B. Slack. 2009. The Geography of Transport Systems, New York: Routledge, $276 \mathrm{pp}$.

20. Sadoddin, A., E. Alvandi and V.B. Sheikh. 2015. Developing a Decision Support System for Participatory and Integrated Management of the Chel-Chai Watershed, Golestan Province, Journal of Watershed Management Research, 6: 124-134 (In Persian).

21. Tacnet, J.M., E. Mermet and S. Maneerat. 2012. Analysis of importance of road networks exposed to natural hazards. Multidisciplinary Research on Geographical Information in Europe and Beyond, Proceedings of the AGILE'2012 International Conference on Geographic Information Science, Avignon, April, 24-27.

22. Usui, H. and Y. Asamim. 2015. An Evaluation of Road Network Patterns Based on the Criteria for Fire-Fighting, European Journal of Geography, 542: 1-22.

23. Vaziri, M. and S. Mostaghimi. 2005. Evaluation of road network of high ways Asia and the Pacific. 2End National Congress on Civil Engineering, Iran University of Science and Technology, 2005-0513, Tehran, pp: 1-6 (In Persian).

24. Xie, F. and D. Levinson. 2007. Measuring the Structure of Road Networks. Geographical Analysis, 39: 336-356.

25. Zhang, Z., J. Bigham, Z. Li and D. Ragland. 2012. Associations between Road Network Connectivity and Pedestrian-Bicyclist Accidents. Transportation Research Board (TRB) $91^{\text {st }}$ Annual Meeting, January 22-26 Washington, D.C.18 pp. 


\title{
Assessment of Road Network Connectivity using Graph Theory in the Chehel- Chai Watershed, Golestan Province
}

\author{
Mohsen Mostafa ${ }^{1}$, Shaban Shataee Jouibary ${ }^{2}$, Majid Lotfalian ${ }^{3}$ and Amir Sadoddin ${ }^{2}$ \\ 1- PhD Student, Gorgan University of Agricultural Sciences and Natural Resources, \\ (Corresponding Author: mohsemmstf@gmail.com) \\ 2- Associate Professor, Gorgan University of Agricultural Sciences and Natural Resources \\ 3- Associate Professor, Sari University of Agricultural Sciences and Natural Resources \\ Received: December 13, 2015 \\ Accepted: April 12, 2016
}

\begin{abstract}
Planning, construction and assessment of road network are among the investigations for infrastructure development at watershed scales. The main aim of this study is assessing the road network connectivity in the Chehel-Chay Watershed, located in Golestan Province using Alpha $(\alpha)$, Beta $(\beta)$, Gamma $(\gamma)$, Eta $(\eta)$, network density and detour indices in graph theory. The available road network map was extracted on 1:25,000 scale, satellite imagery and mapping by GPS. Then the values of indices were calculated for road network that located in forest and nonforest land uses as well as for the whole of the study area. Then, the calculated indices were compared with each other and also with standard index values correspondingly. In forest land use, non-forest land use and whole of the study area the value of calculated indices are: for Alpha index 0.79, 0.14, -.021, for Beta index 0.4, 0.78, 0.56, for Eta index 1.2, 0.64, 1.32, for Gamma index $0.37,0.23,0.19$, for network density index $6.12,27.53,13.12$, and for detour index $0.61,0.81,0.77$, respectively. The results showed that the network connectivity in forest area is suitable but in non- forest area despite of an increase in network density, the network connectivity is inappropriate and for the whole of the study area the network connectivity is considered to be too weak. The network density in forest area is inadequate and in non-forest area network connectivity has been decreased despite of an increase in network density. However, for the whole of the study area the amount t network speed is low due to topographic status and inadequate level of network development. The analysis indicates that the available road network despite of high length of roads constructed in the area and the associated high costs and environmental impacts has not have essential efficiency to connect various land uses in the study area. It is necessary to improve road network based on principles of network connectivity, economic, social and environmental considerations as well as inhabitants' needs in future researches.
\end{abstract}

Keywords: Forest and non- forest land use, Management unite, Network connectivity, Road network, Road segment 